\section{Modelo de transferencia del valor y la experiencia para el turista deportivo}

\section{Value and Experience Model Transfer to the Sports Tourist}

Cristina Aragonés Jericó

Universidad de Valencia y Florida Universitaria cristina.aragones@uv.es

Inés Küster Boluda

Universidad de Valencia

ines.kuster@uv.es

Natalia Vila López

Universidad de Valencia

Natalia.vila@uv.es
Aragonés Jericó, C., Küster Boluda, l., y Vila López, N., (2016). Modelo de transferencia del valor y la experiencia para el turista deportivo.

Revista Internacional de Investigación en Comunicación aDResearch ESIC. No $13 \mathrm{Vol} 13$.

Primer semestre, enero-junio 2016. Págs. 56 a 73 DOI: 13.7263/ADRESIC.013.003 


\section{RESUMEN}

\section{Clasificación JEL:}

L80, L83, M310

Palabras clave:

Turismo deportivo, valor percibido, experiencia, gran evento deportivo, imagen de destino.

El objetivo del estudio es proponer un modelo teórico basado en el comportamiento del turista deportivo y las teorías de patrocinio deportivo. Tras un exhaustivo análisis de la cuestión, este modelo recoge tanto la transferencia de valor y experiencia entre el evento y el patrocinador como los antecedentes del comportamiento del visitante de un gran evento deportivo.

\section{ABSTRACT}

JEL Classification: L80, L83, M310 Key words:

Sport tourism, perceived value, experience, major sport event, destination image.
The aim of this research is to propose a theoretical model based on the behaviour of the sports tourist and sports sponsorship theories. After a thorough analysis of the issue, this model gathers the transfer of value and experience between the event and the sponsor, as well as the previous behaviour of the visitor of a major sporting event. 


\section{Introducción}

En el último informe Familitur publicado, el turismo de eventos deportivos es una de las ra zones que surgen entre las declaradas por los turistas para efectuar un viaje (IET, 2012). Este tipo de turismo está en aumento, ya que el turista deportivo asistiendo al evento vive experiencias únicas e irrepetibles que no podría disfrutar de otro modo. Las ciudades compiten para albergar grandes eventos deportivos, al percibirse como una oportunidad potencial para reposicionar su imagen de destino (Walker, et al., 2013); aumentar el turismo y el desarrollo económico de las comunidades a las que afecta (Mills y Rosentraub, 2013).

Asimismo, las empresas desembolsan grandes cantidades de dinero para patrocinar eventos deportivos; adquiriendo gran importancia frente a otras técnicas de comunicación (Meenaghan y O'Sullivan, 2013). Sin embargo, el patrocinio es un tema que requiere de mayor investigación (Chang y McAleer, 2012). En concreto, al estudio de la transferencia evento-marca patrocinadora (Weed y Bull, 2012; Chang y McAleer, 2012; Mazodier, y Quester, 2014).

Por ello, desde el presente trabajo se postula la necesidad de continuar investigando en este ámbito a fin de poder proporcionar a las empresas recomendaciones para optimizar su gestión. Así, la aportación de este trabajo se trata de una análisis de la cuestión que sean de interés para los gestores empresariales y una aportación en el sentido de ampliación e integración de teorías anteriores.

Se propone como objetivo general del presente artículo: plantear un modelo que considere el comportamiento del turista deportivo y aúne las teorías del patrocinio deportivo, en el marco de un evento deportivo.
El primer objetivo, es el análisis y evaluación del valor-experiencia percibido por el visitante deportivo hacia un evento- marca patrocinadora. El segundo objetivo, es el análisis y evaluación de las variables antecedentes del eje. Fruto del análisis de la literatura y de la revisión de las teorías de patrocinio, estas son: la motivación del turista deportivo, la implicación con el evento, la exposición al evento, la identificación con el equipo y la familiaridad con la marca patrocinadora.

Para alcanzar los objetivos expuestos, se planteó la siguiente estructura: primero se establecen los antecedentes del modelo. A continuación, se propone el modelo de turismo deportivo y, finalmente, se establecen las conclusiones e implicaciones para la gestión.

\section{Antecedentes para un modelo de turismo deportivo}

Para aportar claves que garanticen el éxito de un destino turístico asociado a un gran evento deportivo, se propone un modelo de turismo deportivo. Para ello nos aproximamos conceptualmente al turismo deportivo y al patrocinio deportivo.

\subsection{Turismo deportivo}

El término turismo deportivo fue acuñado por Hall en 1992, defendiéndose como los desplazamientos realizados por razones recreativas para participar u observar actividades deportivas fuera del lugar de residencia habitual. El autor aporta que el turismo deportivo se separa en dos categorías diferenciadas: (i) viajar para participar en un deporte y (ii) viajar para observar un deporte. Por ello, según este autor se podría definir como un viaje, por razones de ocio y no de negocio, para participar u observar actividades deportivas fuera de la residencia habitual. 
A partir de la definición de Hall (1992), los autores Standeven y De Knop (1999) aportan una definición que puede considerarse más difundida y aceptada de turismo deportivo. Los autores concretan que se trata de 'todas las formas activas o pasivas de la actividad deportiva, participando casualmente o de forma organizada, bien sea por razones comerciales o de negocios, pero que necesariamente impliquen un viaje fuera del lugar habitual de residencia o trabajo' (Standeven y De Knop, 1999 p. 12).
Como se recoge en el Cuadro 1, existen otras definiciones sobre el término turismo deportivo y deporte turístico y una extensa polémica sobre la idoneidad de los términos. Ello se debe a que es muy diferente afrontar la definición desde la perspectiva deportiva o desde la perspectiva turística. De ahí que, en los últimos años, el concepto ha evolucionado hasta la inversión de los términos (Latiesa y Paniza, 2006). Asimismo, siguiendo a Latiesa y Paniza (2006) el Cuadro 1 agrupa cada definición dentro de tres concepciones distintas: modelo amplio, modelo intermedio y modelo restrictivo.

\section{Cuadro 1 - Definiciones de turismo deportivo}

\begin{tabular}{|c|c|}
\hline \multirow[t]{2}{*}{ Autores } & Definición \\
\hline & Conceptualización amplia \\
\hline \multicolumn{2}{|c|}{ Actividades que se realizan fuera del lugar habitual de residencia, por motivos competitivos y recreacionales } \\
\hline De Konp (1987) & Turista deportivo: Individuo que participa en deporte durante las vacaciones. \\
\hline \multirow{2}{*}{ Esteve Secall (1991) } & $\begin{array}{l}\text { Turismo deportivo: aquella actividad turística de la que forma parte algún tipo de } \\
\text { práctica deportiva, bien como objetivo fundamental, bien como objetivo complementario. }\end{array}$ \\
\hline & $\begin{array}{l}\text { Deporte turístico: aquellas actividades deportivas susceptibles de comercialización } \\
\text { turística. En la práctica queremos que cualquier actividad deportiva lo sea. }\end{array}$ \\
\hline Kurtzman y Zauhur $(1997,2003)$ & $\begin{array}{l}\text { Turista deportivo: Se trata de un turista seguidor entusiasta de un deporte, ya sea } \\
\text { de forma participativa o no participativa. }\end{array}$ \\
\hline Standeven y De Knop (1999) & $\begin{array}{l}\text { Turismo deportivo: todas las formas activas o pasivas de la actividad deportiva, } \\
\text { participando casualmente o de forma organizada, bien sea por razones comerciales } \\
\text { o de negocios o no, pero que necesariamente implique un viaje fuera del lugar habitual } \\
\text { de residencia o trabajo. }\end{array}$ \\
\hline Neirotti (2003) & $\begin{array}{l}\text { Turismo deportivo: incluye viajar fuera de la residencia habitual para participar en una } \\
\text { actividad deportiva para la recreación o la competición, los viajes para observar deportes, } \\
\text { y los viajes para visitar una atracción deportiva como un pabellón de deportes famoso } \\
\text { o el parque acuático. }\end{array}$ \\
\hline \multicolumn{2}{|r|}{ Conceptualización intermedia } \\
\hline \multicolumn{2}{|c|}{$\begin{array}{l}\text { Turismo motivado a priori por una actividad o evento deportivo para realizar sus desplazamientos recreacionales } \\
\text { o competitivos }\end{array}$} \\
\hline Ruskin (1987) & $\begin{array}{l}\text { Turismo deportivo: Una expresión del comportamiento de las personas en determinados } \\
\text { períodos de tiempo de ocio como las vacaciones, que se realiza en escenarios naturales } \\
\text { especialmente atractivos y en las instalaciones deportivas y físico recreativas al aire libre. }\end{array}$ \\
\hline
\end{tabular}




\begin{abstract}
Hall (1992)

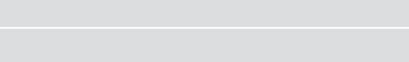

Gammon y Robinson (1997)

Gibson et al. $(1998,2002)$

Latiesa y Paniza (2006)

Hinch y Higham (2011)

Turismo deportivo: desplazamientos realizados por razones recreativas para participar u observar actividades deportivas fuera del lugar de residencia habitual.

Turismo deportivo: personas que viajan fuera de su lugar de residencia habitual y participan activa o pasivamente en deportes competitivos o recreacionales como una actividad secundaria.

Deporte turístico: individuos o grupos que participan activa o pasivamente en deportes competitivos o recreativos durante los viajes que realizan fuera de su residencia habitual.

Turismo deportivo: Ocio y tiempo libre basado en los viajes que temporalmente tienen las personas fuera de sus comunidades de origen para participar en actividades físicas, para observar las actividades físicas, o para venerar lugares de interés relacionados con la actividad física.

Turismo deportivo: aquellas actividades que se desarrollan en un lugar distinto de la residencia habitual y que tienen como objetivo principal la recreación turística y como actividad complementaria vacacional la práctica depo rtiva.

Deporte turístico: actividades que se desarrollan en un lugar distinto de la residencia habitual y que tienen como objetivo principal la actividad deportiva (ejercicio y/o espectáculo) y como actividad complementaria la recreación turística.

Turismo deportivo: viajes basados en el deporte fuera del entorno de residencia habitual por un tiempo limitado; donde el deporte se caracteriza por los conjuntos de reglas únicas, la competencia relacionada con la destreza física y una naturaleza recreativa. Siendo, por tanto algo más que la actividad física.
\end{abstract}

Conceptualización restrictiva

\section{Participación activa o pasiva en eventos deportivos}

\begin{tabular}{|l|l|}
\hline Nogawa, Yamaguchi y Hagi (1996) & $\begin{array}{l}\text { Turista deportivo: Un visitante temporal que permanece al menos } 24 \text { horas en la zona } \\
\text { del evento y cuyo principal objetivo es participar en un evento deportivo siendo el des- } \\
\text { tino una atracción secundaria. }\end{array}$ \\
\hline Weed y Bull (1997) & $\begin{array}{l}\text { Turismo deportivo: Vacaciones que implican actividad deportiva, como espectador } \\
\text { o participante. }\end{array}$ \\
\hline Deery, Jago y Fredline (2004) & $\begin{array}{l}\text { Turista deportivo: participa activa o pasivamente en eventos deportivos competitivos. } \\
\text { El deporte competitivo es el motivo clave para llevar a cabo la experiencia turística. }\end{array}$ \\
\hline Weed y Bull (2012) & $\begin{array}{l}\text { Turismo deportivo: un fenómeno social, económico y cultural que surge por la interac- } \\
\text { ción única entre la actividad, la gente y el lugar. Implica sinergias a la suma entre turismo } \\
\text { y deporte. }\end{array}$ \\
\hline
\end{tabular}

Fuente: Elaboración propia

Ante la diversidad expuesta, se puede afirmar que todas estas definiciones comparten las motivaciones del viaje, capaces de clasificar a un tu- rista como turista deportivo. De ahí la relevancia de ver las principales motivaciones del viaje y clasificar al turista en base a ellas. 
En suma, dentro del turismo de eventos deportivos desde la perspectiva del deporte pasivo, la presente investigación se centra en el comportamiento del espectador deportivo. La literatura existente en este campo revela que, a pesar de que esta línea de investigación ha recibido poca atención (Wann, Melnick, Russell, y Pease, 2001), se observa un creciente interés en el espectador y fan deportivo como campo de estudio académico (Standeven y DeKnop, 1999; Wann, Melnick, Russell, y Pease, 2001; Tapp y Clowes, 2002; Trail y James, 2011; Weed y Bull, 2012; Aiken, Sukhdial, Kahle, y Downing, 2015; Grohs, Reisinger, y Woisetschläger, 2015; Norris, Wann, y Zapalac, 2015). De hecho, durante muchos años, el mayor énfasis en la investigación turística deportiva se ha centrado en los beneficios esperados por la participación en el deporte activo; en contraste con la participación pasiva de espectadores, seguidores y fans (Zillmann, Bryant y Sapolsky, 1979).

\subsection{Patrocinio deportivo}

Es posible definir el patrocinio deportivo desde diversas aproximaciones como muestra el Cuadro 2, donde se relacionan los diferentes autores asociados a las variables contenidas en sus definiciones (Ver Cuadro 2, pág. 62)

De todas las definiciones revisadas, se identifica como definición más completa la de Barreda y Moliner (2004, p. 4). 'El patrocinio deportivo es una herramienta de comunicación en la que se da una provisión de recursos (económicos, fiscales, físicos, humanos) por parte de una o más organizaciones a una o más personalidades deportivas, autoridades deportivas u organismos o códigos deportivos, para permitir al/a los patrocinado/s seguir alguna actividad a cambio de derechos contemplados en términos de la es- trategia de comunicación de marketing del patrocinador, y que pueden ser expresadas en términos de objetivos corporativos, de marketing, de ventas y/o de comunicación, y medidos en términos de conexión entre los objetivos y el resultado deseado en términos de inversión monetaria y no monetaria'.

Por tanto, se entiende por patrocinio deportivo el contrato de patrocinio publicitario en el que el patrocinado es un equipo, un deportista, una actividad o una instalación deportiva. Por ejemplo, en el caso de la Fórmula 1, los contratos son con las escuderías, con los pilotos, con los circuitos, con los grandes premios, etc. En el fútbol, los contratos son con equipos de fútbol, con federaciones de fútbol, asociaciones de fútbol, jugadores de fútbol e instituciones que organizan eventos de este deporte.

Para el caso de la presente investigación, nos parece interesante aproximarnos a la definición del patrocinio deportivo desde un marco general y amplio, considerando todas las variables recogidas en las diversas definiciones analizadas.

Es por ello, que en el presente estudio se propone un modelo global de turismo deportivo que recoge las principales aportación de la literatura, atendiendo al comportamiento del turista deportivo y a partir de las principales teorías de patrocinio deportivo. Estas teorías son los modelos de transferencia afectiva (Pracejus, 2004) y de imagen (Gwinner, 1997) que consideran la transmisión de sentimientos positivos y de asociaciones desde el patrocinado al patrocinador. Asimismo, para el modelo atendemos a otras teorías como son: (i) la teoría del equilibrio (Heider, 1958), ya que frente a asociaciones nuevos se tiende a alterar las percepciones para armonizarlas. (ii) La teoría de la mera exposición ( $\mathrm{Za}$ jonc, 1968; 1980), por la que la exposición repe- 


\section{Cuadro 2: Definiciones de patrocinio deportivo}

\begin{tabular}{|c|c|c|c|c|c|c|c|c|}
\hline & 1 & 2 & 3 & 4 & 5 & 6 & 7 & 8 \\
\hline Abratt y Grobler (1989) & & & $\sqrt{ }$ & & & $\sqrt{ }$ & & \\
\hline Hermanns (1989) & & & & $\sqrt{ }$ & & $\sqrt{ }$ & & \\
\hline Sleight (1989) & & & $\sqrt{ }$ & & & $\sqrt{ }$ & & \\
\hline Berrett (1993) & $\sqrt{ }$ & & & & $\sqrt{ }$ & & & \\
\hline Wilmshurst (1993) & $\sqrt{ }$ & $\sqrt{ }$ & & & & $\sqrt{ }$ & & \\
\hline Dibb, Simkin, Pride y Ferrell (1994) & $\sqrt{ }$ & $\sqrt{ }$ & & & & & & \\
\hline Adcock, Bradfield, Aalborg y Ross (1995) & & & $\sqrt{ }$ & & & & $\sqrt{ }$ & \\
\hline Jobber (1995) & $\sqrt{ }$ & & & & & $\sqrt{ }$ & & \\
\hline Siegel (1996) & & & & & & & $\sqrt{ }$ & \\
\hline Shimp (1997) & & & & & & & $\sqrt{ }$ & \\
\hline Shank y Beasley (1998) & $\sqrt{ }$ & & & & $\sqrt{ }$ & & & \\
\hline Van Heerden (2001) & & & & & $\sqrt{ }$ & & $\sqrt{ }$ & $\sqrt{ }$ \\
\hline Barreda y Moliner (2004) & & & & & $\sqrt{ }$ & & $\sqrt{ }$ & $\sqrt{ }$ \\
\hline \multicolumn{9}{|l|}{$1=$ Relación financiera } \\
\hline \multicolumn{9}{|l|}{$2=$ Relación material } \\
\hline \multicolumn{9}{|l|}{3 = Relación comercial } \\
\hline \multicolumn{9}{|l|}{$4=$ Relación contractual } \\
\hline \multicolumn{9}{|c|}{$5=$ Objetivos empresariales y de marketing } \\
\hline \multicolumn{9}{|c|}{$6=$ Beneficio para el patrocinador $y / o$ el patrocinado } \\
\hline \multicolumn{9}{|c|}{$7=$ Forma de promoción /estrategia de comunicación } \\
\hline $8=$ Provisión de recursos del patrocina & pat & & & & & & & \\
\hline
\end{tabular}

tida a un estímulo con el que se identifica supone les a los que se pertenece. (iv) La teoría de la seel conocimiento al mismo. (iii) La teoría de la ñal (Spence, 1973 y Ross, 1977) que afirma que identidad social (Tajfel y Turner, 1979), en la las señales de marketing influyen en la codificaque se establecen conexiones con grupos sociación de la información del consumidor. Y por 
último, (v) la teoría de la atribución (Heider, 1958; Jones y Davis, 1965; Kelley, 1973) que defiende la relación directa entre las motivaciones y sus actitudes y comportamientos.

En base a lo expuesto, para el desarrollo efectivo de acciones de marketing y la toma de decisiones de los agentes que intervienen en el sector del turismo de eventos deportivos y de las posibles organizaciones patrocinadoras, resulta de gran utilidad conocer la influencia de la transferencia del evento patrocinado hacia la marca patrocinadora.

\section{Planteamiento de un modelo de turismo deportivo}

\subsection{Eje central: valor percibido y expe- riencia, transferencia del evento a la marca}

El modelo se basa en los Modelos de Transferencia (Gwinner, 1997). La asociación entre evento y marca conducen al visitante al evento a trasladar las percepciones del evento a la marca que lo patrocina (Meenaghan, 2001; Grohs y Reisinger, 2005; Küster, Vila, Aldás y Rodríguez, 2009; Gijsenberg, 2014; Mazodier y Quester, 2014; Carrillat, Solomon, y d'Astous, 2015; Cornwell, 2015; Thomas, 2015). En concreto, el modelo propuesto se aplica a la transferencia del valor percibido y a la transferencia de la experiencia.

Estos dos conceptos, estrechamente ligados al comportamiento del turista de este tipo de eventos, cobran cada vez más peso en la disciplina de marketing debido a la sofisticación del consumidor y el aumento de la competencia, entre otros. De hecho, en el ámbito del turismo, el estudio del comportamiento del turista ha mostrado la relevancia del valor percibido y la experiencia en el proceso de toma de decisiones.
En primer lugar, el valor percibido es un factor decisivo para el desarrollo empresarial, porque constituye el eje fundamental sobre el que se sustenta el desarrollo de las ventajas competitivas (Lee, et al., 2004; Brodie, et al., 2009; CheHui, Wen, y Chung-Cheng, 2011), derivado de la importancia que tiene en el comportamiento del consumidor. En base a ello, el valor percibido se ha convertido en el eje central de las actividades de marketing. En el ámbito de aplicación de la presente investigación, el valor percibido requiere especial atención porque se ve influenciado por el evento y la marca patrocinadora (Koo, et al., 2006; Aldás, et al., 2008). Así, es posible establecer que el proceso de transferencia entre el evento y la marca se aprecia en lo relativo al valor percibido (Koo, et al., 2006).

En segundo lugar, la experiencia vivida es clave en el sector turístico, como respuesta a una estimulación inducida por algo o alguien externo (Alcaide y Merino, 2011). Este punto ha cobrado relevancia creciente desde el trabajo pionero de Pine y Gilmore (1999) sobre 'La economía de la experiencia', y los primeros artículos científicos basados en la experiencia turística (Otto y Ritchie, 1996). Holbrook y Hirschman (1982) proponen pasar del 'mundo de producto' al 'mundo de la experiencia', entendiendo el comportamiento del turista, no sólo como de procesamiento de la información, sino esencialmente experiencial, sin ignorar el carácter placentero de las actividades de ocio y la importancia de los placeres sensoriales, los sueños, el goce estético, y las respuestas emocionales. Por tanto, el enfoque experiencial implica identificar las emociones que el individuo siente tras una experiencia (Lenderman, 2008). En la actualidad, no basta con ofrecer productos y servicios turístico-deportivos, sino que hay que ofre- 
cer experiencias 'intensas y reales' (Alfaro, 2011). Así, es necesario conocer la manera en que el turista piensa y siente en el momento de la experiencia, como la recordará en el futuro, y cómo va a contribuir a la satisfacción total de la actividad.

A partir de esta idea y en base a la clasificación de tipos de experiencias propuesta por Schmitt (1999), la presente investigación se ha basado en las emociones para aproximarse a la experiencia hacia el evento patrocinado, y en las actitudes para conocer la experiencia hacia la marca patrocinadora. Se asume así, que los asistentes al evento deportivo, por su relación con el mismo y por el ambiente físico, pueden generar diversas emociones (Bitner, 1992). Mientras que, como consecuencia del patrocinio, dichos asistentes al evento realizan asociaciones mentales, que se traducirá en una determinada actitud y comportamiento hacia la marca patrocinadora (Meenaghan, 2001).

Así, respecto al objetivo principal, y en base a los Modelos de Transferencia, postulamos la transferencia del evento a la marca, tanto del valor percibido como de la experiencia. Del mismo modo, según lo argumentado en la Teoría de la Atribución y en los modelos del comportamiento del turista, planteamos el efecto de la experiencia sobre el valor percibido.

\subsection{Antecedentes del comportamiento del visitante de un evento deportivo.}

Atendiendo al segundo objetivo de la investigación, en este apartado se recogen los cinco antecedentes del eje del modelo: motivaciones, implicación con el evento, exposición al evento, identificación con el equipo, familiaridad con la marca.

Respecto a las motivaciones, excepto en los viajes por motivos de trabajo, raramente las mo- tivaciones del turista están influidas por un sólo motivo, la decisión de emprender un viaje y la elección de un destino, dándose varias motivaciones combinadas (Trail y James, 2011). De ahí la necesidad de tener en cuenta aspectos racionales e irracionales, conscientes e inconscientes, externos e internos, etc. Así, la elección del tipo de vacaciones y del destino viene representada por un compromiso entre las múltiples motivaciones que se interrelacionan entre sí y las posibilidades que el destino ofrece, pero en algunas ocasiones es posible encontrar un motivo predominante, como por ejemplo la asistencia a un evento deportivo (como es el caso de la presente investigación). Por lo que respecta a la motivación para realizar turismo de eventos deportivos, la revisión de la literatura muestra diversas motivaciones pertenecientes a dimensiones: psicológicas (estética / placer estético, drama / estrés, escapar, atracción física, entretenimiento, excitación, suspense); socio-culturales (interacción familiar y social, interacción social / grupo de afiliación, conexiones culturales, adquisición de conocimiento, habilidades físicas, factores económicos, modelos de rol, ambiente saludable, apoyo a las oportunidades de las mujeres, aspectos técnicos); y de pertenencia social (logro indirecto / autoestima, interés en el deporte, interés en el equipo, interés en el jugador, orgullo nacional, conexiones tribales).

De este modo, a partir de los estudios previos, y tomando como línea argumental lo postulado en la Teoría de la Atribución, planteamos que a más motivación más identificación con el equipo, y para el evento, más valor percibido, satisfacción y comportamiento futuro.

Por otro lado, se ha propuesto la implicación con el evento como un antecedente al considerarlo un factor clave en el proceso de persuasión 
del patrocinio, analizado en diversas investigaciones de espectadores deportivos (Fink, Trail y Anderson, 2002; Bodet, Bernache-Assollant y Kada, 2011). Además de representar un aspecto determinante para que el consumidor se vea expuesto al evento, también posee un efecto significativo en la identificación con el equipo (o deportista) y el conocimiento de la marca patrocinadora.

Por todo ello, se postula que la implicación con el evento se asocia positivamente con la exposición, la identificación y el conocimiento de marca, tomando, para ello, como premisa la Teoría de la Identidad Social.

Otro antecedente considerado en esta investigación es la exposición al evento, importante para que el patrocinio se convierta en una herramienta de comunicación efectiva. El visitante al evento deportivo está disfrutando de un espectáculo que encaja con sus patrones de ocio, por lo que podemos afirmar que el patrocinio se convierte en una herramienta de comunicación más efectiva que otras. Así, y en base a la argumentación defendida por la Teoría de la Mera Exposición, nos centramos en la exposición al evento y su influencia tanto en el valor percibido del evento como en la experiencia con el evento.
La identificación con el equipo también se asume como antecedente a la transferencia entre evento y marca patrocinadora, por entenderse como la percepción de la conectividad de los espectadores a un equipo y la experiencia de los fallos y logros del equipo como si fueran propios (Ashforth y Mael, 1989). En este contexto, y en relación con los resultados alcanzados en estudios previos, basados en la Teoría de la Identidad Social, es posible plantear que conforme más identificación más experiencia con el evento y la marca, y más conocimiento y satisfacción con la marca.

Adicionalmente, podemos asumir que la familiaridad con la marca en términos de patrocinio, entendida como la cantidad de asociaciones que se encuentran en la mente de los consumidores sobre el evento patrocinado y el patrocinador, es un antecedente y punto de partida importante para explicar la identificación del patrocinio y transferencia de percepciones hacia la marca. Por ello, a partir de las premisas recogidas en la Teoría de la Señal y la Teoría del Equilibrio, es posible plantear que la familiaridad con la marca supone un mayor valor de marca.

Con objeto de facilitar la lectura y la interpretación de todas las hipótesis de investigación, se construye la Tabla 1.

\section{Tabla 1 • Resumen de las hipótesis y objetivos y subobjetivos de investigación}

Objetivo 1: Análisis y evaluación del vínculo valor-experiencia percibidos por el visitante deportivo hacia un evento patrocinado - marca patrocinadora.

Subobjetivos:

1.1 Transferencia del valor percibido del evento hacia el valor percibido de la marca que lo patrocina.

1.2 Transferencia de la experiencia con el evento hacia la experiencia con la marca que lo patrocina.

1.3 Efecto de la experiencia con el evento sobre el valor percibido del evento.

1.4 Efecto de la experiencia con la marca patrocinadora sobre el valor percibido de la marca patrocinadora. 


\section{Tabla 1 • Resumen de las hipótesis y objetivos y subobjetivos de investigación (continuación)}

Hipótesis relativas al eje central del modelo

H1: El valor percibido del evento afectará al valor percibido de la marca.

H2: La experiencia que se tenga con un evento afectará a la experiencia que se tenga con una marca.

H3: La experiencia tendrá un efecto sobre el valor percibido.

H3.1: La experiencia con el evento tendrá un efecto sobre el valor percibido del evento.

H3.2: La experiencia con la marca tendrá un efecto sobre el valor percibido de la marca.

Objetivo 2: Análisis y evaluación de las variables antecedentes de la transferencia valor percibido-experiencia. Subobjetivos:

2.1 Análisis y evaluación de la motivación del turista deportivo.

2.2 Análisis y evaluación de la identificación con el equipo.

2.3 Análisis y evaluación de la implicación con el evento.

2.4 Análisis y evaluación de la exposición al evento.

2.5 Análisis y evaluación de la familiaridad con la marca patrocinadora.

Hipótesis relativas a las variables antecedentes

H4: A más motivaciones del turista deportivo:

H4.1: Mayor será la identificación con el equipo.

H4.2: Mayor será el valor percibido del evento.

H5: La implicación con el evento está positivamente asociada con:

H5.1: Una mayor exposición al evento.

H5.2: La identificación con el equipo.

H6: La exposición al evento patrocinado se asocia positivamente con:

H6.1: El valor percibido del evento

H6.2: La experiencia del espectador con el evento.

H7: La identificación con el equipo se asocia positivamente con:

H7.1: La experiencia con el evento.

H7.2: La experiencia con la marca.

H8: La familiaridad con la marca se asocia positivamente con el valor percibido de marca. 


\section{Figura 1 - Modelo de patrocinio deportivo propuesto}

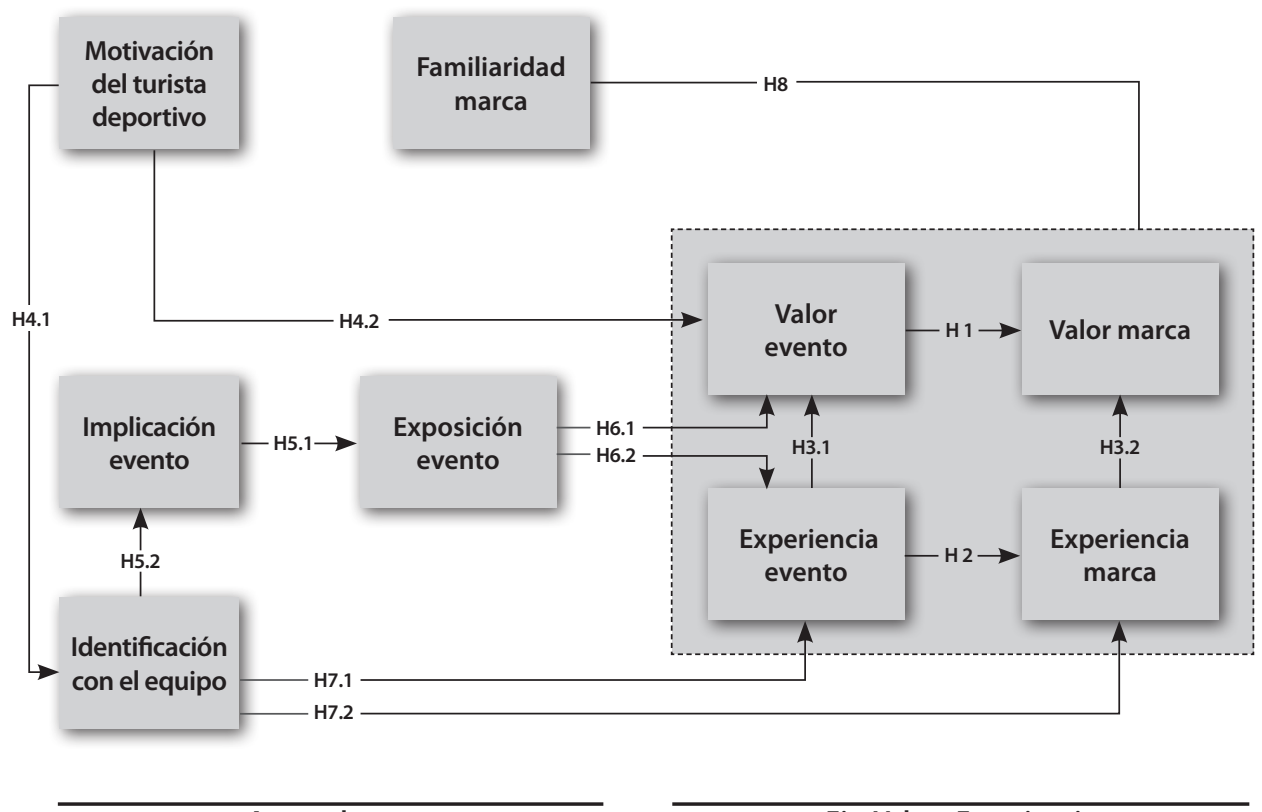

Antecedentes

Eje: Valor - Experiencia

Llegados a este punto, la suma de objetivos e hipótesis expuestos previamente, da como resultado el modelo de patrocinio deportivo que se muestra en la Figura 1.

\section{Conclusiones, implicaciones, limitaciones y futuras líneas}

En esta investigación se ha presentado una propuesta de modelización y medida con la que poder avanzar en el campo de la investigación del turismo de eventos deportivos. Para ello se ha desarrollado el modelo y se han identificado los principales constructos que lo componen. Las conclusiones aquí planteadas se realizan en función de la amplia revisión bibliográfica realizada.
Es posible concluir que los poderes públicos organizan eventos tratando de aumentar el atractivo de sus ciudades y crear imagen de destino. Al tiempo que los asistentes de eventos deportivos vive experiencias únicas.

En primer lugar, destaca lo relevante que ha sido la investigación de la transferencia experiencia-valor percibido a nivel de evento y de marca. Ambos conceptos (valor percibido y experiencia) cada vez cobran más peso en la disciplina de marketing debido a la sofisticación del consumidor y el aumento de la competencia, entre otros. De hecho, en el ámbito del turismo, el estudio del comportamiento del turista ha 
mostrado la relevancia del valor percibido y la experiencia en el proceso de toma de decisiones.

En segundo lugar, la revisión de la literatura realizada respecto al concepto del turismo deportivo, permite observar como la motivación es una herramienta fundamental para explicar el comportamiento del turista deportivo. Aquí es posible concluir que juega un papel relevante el desarrollo de la comercialización turística y el marketing.

En tercer lugar, es posible enmarcar el patrocinio en las estrategias empresariales de relaciones públicas y comunicación corporativa. Así, concluimos que el patrocinio deportivo es la relación financiera, material, comercial y contractual que implica la provisión de recursos del patrocinador al patrocinado en busca de la consecución de objetivos empresariales y de marketing gracias a la promoción y a la estrategia de comunicación utilizada, persiguiendo el beneficio mutuo, tanto para el patrocinador como para el patrocinado.

En cuarto lugar, la revisión de la literatura nos permite concluir que la asociación evento - marca lleva al visitante a trasladar sus percepciones. Considerándose los antecedentes de dicha asociación se relacionan las siguientes conclusiones.

Por lo que respecta a la motivación para realizar turismo de eventos deportivos, la revisión de la literatura muestra diversas motivaciones pertenecientes a dimensiones: psicológicas; socioculturales y de pertenencia social.

Por otro lado, nos hemos aproximado a la implicación con el evento como un antecedente al considerarlo un factor clave en el proceso de persuasión del patrocinio. Además de representar un aspecto determinante para que el consumidor se vea expuesto al evento, también posee un efecto significativo en la identificación con el equipo y el conocimiento de la marca patrocinadora.
Otro antecedente considerado en esta investigación ha sido la exposición al evento, importante para que el patrocinio se convierta en una herramienta de comunicación efectiva. El visitante al evento deportivo está disfrutando de un espectáculo que encaja con sus patrones de ocio, por lo que podemos concluir que el patrocinio se convierte en una herramienta de comunicación más efectiva que otras.

La identificación con el equipo también se ha considerado como antecedente a la transferencia entre evento y marca patrocinadora, por entenderse como la percepción de la conectividad de los espectadores a un equipo y la experiencia de los fallos y logros del equipo como si fueran propios (Ashforth y Mael, 1989).

Adicionalmente, podemos concluir que la familiaridad con la marca en términos de patrocinio ha sido un antecedente y punto de partida importante para explicar la identificación del patrocinio y transferencia de percepciones hacia la marca.

A continuación se recogen las implicaciones y recomendaciones aportadas para los gestores y responsables de la gestión de eventos en las ciudades.

En primer lugar, es importante que los destinos turísticos puedan adaptarse a las exigencias de los turistas que practican el turismo de eventos deportivos.

En segundo lugar, el turismo de eventos deportivos, se prevé que siga creciendo, permitiendo a los destinos diferenciarse y aumentar su competitividad a un nivel internacional. Asimismo, los eventos deportivos facilitan a la ciudad anfitriona una excelente campaña de imagen y atraen a un importante número de visitantes, tanto turistas como excursionistas. Por ello, debería promoverse la celebración de este tipo de 
eventos, pero sin olvidar la integración de la comunidad anfitriona.

En tercer lugar, si los gestores del evento deportivo contemplan las diferentes motivaciones del visitante podrán ver mejorado el valor percibido que tiene respecto al evento. De esta forma, será más probable que el visitante recomiende el evento entre sus conocidos y que asista a próximas ediciones.

Antes de cerrar esta trabajo, es preciso indicar las limitaciones que han condicionado su desarrollo y las futuras líneas de investigación que emanan tanto de los aspectos no tratados en la presente investigación como de los resultados y objetivos logrados, que permitirán una mayor profundización en la aplicación del turismo deportivo como turismo de experiencias e imagen de las ciudades.

Algunas limitaciones surgen de la propia delimitación del estudio, tanto en el ámbito de aplicación como en aspectos relacionados con la determinación final del modelo propuesto. Es por ello que de las mismas podemos obtener futuras líneas de investigación. En primer lugar, resulta especialmente interesante incluir variables moderadoras al modelo, en concreto consideramos pertinente conocer la moderación que pueden ejercer la congruencia entre evento y marca y la nacionalidad del visitante.

En segundo lugar, planteamos analizar la moderación de la nacionalidad, porqué a pesar de que la investigación cross-cultural se remonta varias décadas atrás, algunos autores destacan que, sorprendentemente, el efecto de la cultura en la toma de decisiones de los consumidores está poco investigado, especialmente en Europa (Salciuviene, Auruskeviciene y Lideka, 2005).

Por último, el modelo se ha centrado en sus antecedentes, pero una futura línea de investigación podría abordar también los efectos de la transferencia en materia de valor percibido y experiencia. 


\section{Bibliografía}

Abratt, R., \& Grobler, P. S. (1989). The evaluation of sports sponsorships. International Journal of Advertising, 8 (4), 351362.

Adcock, D.; Bradfield, R.; Halborg, A. \& Ross, C (1995). Marketing principles and practice. Second Edition. Pitman Publising, London, UK.

Aiken, D., Sukhdial, A., Kahle, L., \& Downing, J. A. (2015). Linking Fan Values and Sponsorship Effectiveness: The Case of Old School Values. Sport Marketing Quarterly, 24(1), 56.

Alcaide Casado, J. C., \& Merino Sanz, M. J. (2011). Comunicación experiencial y sensorial: algunos ejemplos de aplicación. Harvard Deusto Business Review, (198), 62-79.

Aldás, J.; Bachmann, F; Dalli, D.; Hennings, N.; Küster, I.; Moscarola, J.; Vila, N.; \& Zuccella, A. (2008). «Cross-National Differences in Customer Evaluation of Sponsor-Event Association». 7th International Congress of Marketing Trends, Venecia, Italia.

Alfaro García, E. (2011). Customer Experience Management (parte I). Vender más en tiempos de crisis. MK. Marketing y Ventas para Directivos, (264), 20-29.

Ashforth B. \& Mael, F. (1989). «Social identity theory and the organization». The Academy of Management Review, January, Vol. 14, núm. 1, p. 20-39.

Barreda Tarrazona, R. \& Moliner Tena, M. A. (2004): «Respuesta cognitiva al patrocinio deportivo. Un estudio empírico en el fútbol de la primera división española». XVIII Congreso Anual y XIV Congreso Hispano-Francés de AEDEM. Ourense: 02-06-2004.

Bennett, G., Ferreira, M., Lee, J., Polite, F. (2009). The role of involvement in sports and sport spectatorship in sponsor's brand use: The case of Mountain Dew and action sports sponsorship. Sport Marketing Quarterly, 18(1), 14-24.

Berrett, T. (1993). The Sponsorship of Amateur Sport-Government, National Sport Organization, and Corporate Perspectives. Loisir et Société/Society and Leisure, 16(2), 323346.

Bitner, M. J. (1992): Servicescapes: the impact of physical surroundings on customers and employees. The Journal of Marketing, 57-71.

Bouchet, P., Bodet, G., Bernache-Assollant, I., \& Kada, F. (2011): Segmenting sport spectators: Construction and pre- liminary validation of the Sporting Event Experience Search (SEES) scale. Sport management review, 14(1), 42-53.

Brodie, R. J., Whittome, J. R., \& Brush, G. J. (2009). Investigating the service brand: A customer value perspective. Journal of Business Research, 62(3), 345.

Carrillat, F. A., Solomon, P. J., \& d'Astous, A. (2015). Brand Stereotyping and Image Transfer in Concurrent Sponsorships. Journal of Advertising, 1-15.

Cornwell, T. B. (2015). Sponsorship-Linked Marketing. Routledge Handbook of Theory in Sport Management.

Chang, C. L., \& McAleer, M. (2012). Citations and impact of ISI tourism and hospitality journals. Tourism Management Perspectives, 1, 2-8.

Che-Hui, L., Wen, M., \& Chung-Cheng, W. (2011). Investigating the relationships among E-service quality, perceived value, satisfaction, and behavioral intentions in Taiwanese online shopping. Asia Pacific Management Review, 16(3), $\mathrm{n} / \mathrm{a}$.

Chen, K. K., Zhang, J. J. (2011). Examining consumer attributes associated with collegiate athletic facility naming rights sponsorship: Development of a theoretical framework. Sport Management Review, 14(2), 103-116.

Deery, M., Jago, L. \& Fredline, L. (2004): Sport tourism or event tourism: are they one and the same? Journal of Sport and Tourism, 9(3), 235-245.

Dibb, S., Simkin, L., Pride, W. M., \& Ferrell, O. C. (2005). Marketing: Concepts and strategies (p. 850). Houghton Mifflin.

Esteve Secall, R. (1991): «Análisis Teórico de las Relaciones Entre el Turismo y el Deporte, Referencia Especial a Andalucía», En I Jornadas Sobre Turismo y Deporte, Consejería de Cultura, Instituto Andaluz del Deporte.

Fink, J. S., Trail, G. T., \& Anderson, D. F. (2002): «Environmental factors associated with spectator attendance and sport consumption behavior: gender and team differences». Sport Marketing Quarterly, 11(1), 8-19.

Gammon, S., \& Robinson, T. (1997): 'Sport And Tourism: A Conceptual Framework', Journal of Sport Y Tourism,4:3, 11-18

Gibson, H., Attle, S. \& Yiannakis, A. (1998): «Segmenting the sport tourist market: a lifespan perspective», Journal of Vacation Marketing, Vol. 4, pp. 52-64. 
Gibson, H.J., Willming, C., \& Holdnak, A. (2002): 'We're Gators ... not just Gator fans': serious leisure and University of Florida football. Journal of Leisure Research, 34(4), $397-$ 426.

Gijsenberg, M. J. (2014). Comment: Measuring marketing effectiveness around major sports events: A comparison of two studies and a call for action. International Journal of Research in Marketing, 31(1), 30-32.

Grohs, R., \& Reisinger, H. (2005). Image transfer in sports sponsorships: An assessment of moderating effects. International Journal of Sports Marketing and Sponsorship, 7(1), 42.

Grohs, R., Reisinger, H., \& Woisetschläger, D. M. (2015). Attenuation of negative sponsorship effects in the context of rival sports teams' fans. European Journal of Marketing, 49(11/12).

Gwinner, K. (1997). A model of image creation and image transfer in event sponsorship. International marketing review, 14(3), 145-158.

Hair, J. F., Black, W. C., Babin, B. J., Anderson, R. E., \& Tatham, R. L. (2009). Multivariate data analysis (Vol. 7). Upper Saddle River, NJ: Prentice Hall.

Hall, C.M. (1992). «Adventure, Sport and Helth», En Hall, C.M. Y B. Weiler (Eds.), Special Interest Tourism, Pluto Press, Londres.

Heider, F. (1958). The psychology of interpersonal relations. New York: Wiley

Hinch, T., \& Higham, J. (2011). Sport tourism development (Vol. 13). Channel View Books.

Holbrook, M. B., \& Hirschman, E. C. (1982). The experiential aspects of consumption: consumer fantasies, feelings, and fun. Journal of consumer research, 132-140.

Hutchinson, J., Lai, F., \& Wang, Y. (2009). Understanding the relationships of quality, value, equity, satisfaction, and behavioral intentions among golf travelers. Tourism Management, 30(2), 298-308.

Instituto de Estudios Turísticos (IET), (2012). Informe Anual 2011 Movimientos turísticos de los españoles (FAMILITUR). Retrieved May 3, 2013, from http://www.iet.tourspain.es/es-ES/estadisticas/familitur/Anuales/Informe\%20 anual $\% 20 \mathrm{de} \% 20$ Familitur.\%20A\%C3\%Blo\%202012.pdf

Jobber, D. (1995). Principles and practice of marketing. McGraw-Hill Book Company, London, UK.
Jones, E. E., \& Davis, K. E. (1965). From acts to dispositions the attribution process in person perception. Advances in experimental social psychology, 2, 219-266.

Kelley, H. H. (1973). The processes of causal attribution. American psychologist, 28(2), 107.

Koo, G. Y., Quarterman, J., \& Flynn, L. (2006). Effect of perceived sport event and sponsor image fit on consumers cognition, affect, and behavioral intentions. Sport Marketing Quarterly, 15(2), 80-90

Kurtzman, J. \& Zauhar J. (1997), «A wave in time, the sport tourism phenomena», Journal of Sport Tourism, 4, (2)

Kurtzman, J. \& Zauhar J. (2003) A wave in time: the sport tourism phenomenon. Journal of Sport Tourism, 8(1): 35-47.

Küster, I., Vila, N., Aldás, J., \& Rodríguez, C. (2009). Efecto del Patrocinio de la Copa América en las percepciones de Luis Vuitton: una perspectiva internacional. Universia Business Review, (22), 40-55.

Latiesa, M., \& Paniza, J. L. (2006). Turistas deportivos. Una perspectiva de análisis. Revista internacional de sociología 64(44), 133-149

Lee, E. J., Uniremidy, C. A., \& Overby, J. W. (2004). Creating value for online shoppers: implications for satisfaction and loyalty. Journal of Consumer Satisfaction, Dissatisfaction and Complaining Behaviour, 17, 54-68.

Lee, J., Lee, C., \& Choi, Y. (2011). Examining the role of emotional and functional values in festival evaluation. Journal of Travel Research, 50(6), 685.

Lenderman, M. (2008). Marketing Experiencial: La revolución de las marcas. ESIC Editorial.

Liang, B., \& Cherian, J. (2010). Cross-cultural differences in the effects of abstract and concrete thinking on imagery generation and ad persuasion. Journal of International Consumer Marketing, 22(2), 187-198.

Mazodier, M., \& Quester, P. (2014). The role of sponsorship fit for changing brand affect: A latent growth modeling approach. International Journal of Research in Marketing, 31(1), $16-29$

Meenaghan, T. (2001). «Sponsorship and advertising: a comparison of consumer perceptions». Psychology \& Marketing. Vol. 18, N. 2, pp. 191-215.

Meenaghan, T., \& O'Sullivan, P. (2013). Metrics in Sponsorship Research-Is Credibility an Issue?. Psychology \& Marketing, 30(5), 408-416. 
Mills, B. M., \& Rosentraub, M. S. (2013). Hosting megaevents: A guide to the evaluation of development effects in integrated metropolitan regions. Tourism Management, 34, 238-246.

Neirotti, L. D. (2003) An Introduction to Sport and Adventure Tourism. In Hudson, S. (Ed.) Sport and Adventure Tourism, Haworth: Binhampton, NY, 1-25

Nogawa, H., Yamaguchi, Y., \& Hagi, Y. (1996). «An empirical research study on Japanese sport tourism in sport for all events: case studies of a single night even and a multiple night event», Journal of Travel Research, 46-54.

Norris, J. I., Wann, D. L., \& Zapalac, R. K. (2015). Sport fan maximizing: following the best team or being the best fan?. Journal of Consumer Marketing,32(3), 157-166.

Otto, J. E., \& Ritchie, J. R. (1996). The service experience in tourism. Tourism Management, 17(3), 165-174.

Pine J., \& Gilmore J. (1999). The Experience Economy: Work is Theatre $\&$ Every Business a Stage. Harvard Business School Press: Boston.

Pracejus, J. W. (2004): «Seven psychological mechanisms through which sponsorship can influence consumers». Sports marketing and the psychology of marketing communication, 175-89.

Prebensen, N. K., Woo, E., Chen, J. S., \& Uysal, M. (2013). Motivation and involvement as antecedents of the perceived value of the destination experience. Journal of Travel Research, 52(2), 253.

Ramirez, D., Laing, J., Mair, J. (2013). Exploring Intentions to Attend a Convention: A Gender Perspective. Event Management, 17(2), 165-178.

Razi, M. A., Siddiquei, A. N., Awan, H. M., \& Bukhari, K. (2012). Relationship between service quality, perceived value, satisfaction and revisit intention in hotel industry. Interdisciplinary Journal of Contemporary Research in Business, 4(8), 788-805.

Ross, S. A. 1977. The determination of financial structure: The incentive signaling structure. Bell Journal of Economics, 8: 23-40.

Salciuviene, L., Auruskeviciene, V., \& Lydeka, Z. (2005). An assessment of various approaches for cross-cultural consumer research. Problems and Perspectives in Management, 3(3), 147-159.
Sánchez, J., Callarisa, L., Rodríguez, R. M., \& Moliner, M. A. (2006). Perceived value of the purchase of a tourism product. Tourism Management, 27(3), 394-409.

Schmitt, B. (1999): «Experiential marketing». Journal of Marketing 15: 53-67.

Shank, M. D., \& Beasley, F. M. (1998). «Fan or fanatic: Refining a measure of sports involvement». Journal of sport behavior.

Shimp, T. (1997). Advertising, Promotion, and Supplemental Aspects of Integrated Marketing Communications, 4th edn. New York: Dryden Press.

Sleight, S. (1989). Sponsorship: what it is and how to use it. Maidenhead, McGraw-Hill.

Spence, M. (1973). Job market signaling. Quarterly Journal of Economics, 87: 355-374.

Standeven, J., P. \& De Know (1999), Sport Tourism, Human Kinetics.

Tapp, A. \& Clowes, J. (2002): «From 'carefree casuals' to 'professional wanderers': Segmentation possibilities for football supporters», European Journal of Marketing, Vol. 36, No 11/12, pp. 1248-1269.

Thomas, R. J. (2015). Out with the old and in with the new: a study of new kit sponsorship and brand associations in the Barclays Premier League. Journal of Product $\&$ Brand Management, 24(3), 229-251.

Trail, G. T., \& James, J. D. (2011). Sport consumer behavior. Seattle, WA: Sport Consumer Research Consultants.

Van Heerden, C. H. (2001). «Factors affecting decision-making in South African sport sponsorships». Tesis Doctoral. University of Pretoria. South Africa.

Walker, M., Kaplanidou, K., Gibson, H., Thapa, B., Geldenhuys, S., \& Coetzee, W. (2013). «Win in Africa, With Africa»: Social responsibility, event image, and destination benefits. The case of the 2010 FIFA World Cup in South Africa. Tourism management, 34, 80-90.

Wann, D. L., Melnick, M. J., Russell, G. W. \& Pease, D. G. (2001). Sport Fans: The Psychology and Social Impact of Spectators. London: Routledge.

Weed, M. E., \& Bull, C. J. (1997). Integrating sport and tourism: A review of regional policies in England. Progress in Tourism and Hospitality Research,3(2), 129-147.

Weed, M., \& Bull, C. (2012). Sports Tourism 2e. Routledge. 
Wilmshurst, J. (1993). Below-the-line promotion. Butterworth-Heinemann Ltd. Oxford, UK.

Woodruff, R. B. (1997). Customer value: The next source for competitive advantage. Academy of Marketing Science. Journal, 25(2), 139-153.

World Travel \& Tourism Council. (2013). The Authority on World Travel \& Tourism. Retrieved May 6, 2013, from http:// www.wttc.org/site_media/uploads/downloads/ world2013_1.pdf
Zajonc, R. B. (1968). Attitudinal effects of mere exposure. Journal of personality and social psychology, 9(2p2), 1.

Zajonc, R. B. (1980). Feeling and thinking: Preferences need no inferences.American psychologist, 35(2), 151.

Zillmann, D., Bryant, J., \& Sapolsky, B. S. (1989). Enjoyment from sports spectatorship. Sports, games, and play: Social and psychological viewpoints, 2, 241-278. 